\title{
Follicular Response by using Letrozole alone or combination of Letrozole and Gonadotrophins in patients stimulated for IUI
}

\author{
RAHMAN $F^{1}$, MAHMUD N² ${ }^{2}$ CHOWDHURY TA ${ }^{3}$, RAHMAN RRB ${ }^{4}$, BEGUM B $^{5}$
}

\begin{abstract}
:
IUI with controlled ovarian hyperstimulation is a well known means to achieve pregnancy in unexplained and mild to moderate male factor infertility, which is also inexpensive, easy to perform as office procedure. The success rate is high.

Objective: To assess the follicular response by Letrozole alone or by combination of Letrozole and Gonadotrophins in patients stimulated for IUI.

Method: Prospective observational type of study.

Place: CARE BIRDEM

Time: $10^{\text {th }}$ May $2010-31^{\text {st }}$ December 2010

Population: All the OPD patients of CARE who were selected for IUI during this period. Patients were divided into two age groups ( $<30 \mathrm{yrs}$ and $>30 \mathrm{yrs}$ )

Procedure: Ovulation induction was done by letrozole in one group, and combined letrozole and gonadotrophins in another group. Monitoring was done by TVS for 3 cycles.

Result: Mean follicular number was 1.28 \pm 0.94 in letrozole and $3.37 \pm 0.87$ in combined letrozole and gonadotrophins. No. of follicles $>18 \mathrm{~mm}$ was $12 \mathrm{pts}(3.54 \pm 1.63)$ in letrozole, and 50 pts (3.44 \pm 1.93 ) in combined letrozole and gonadotrophins. Pregnancy/cycle was 12/77 (5.19\%) in letrozole and 28/65 (14.3\%) in combined letrozole and gonadotrophins.

Conclusion: Number of mature follicle and pregnancy rate is higher in combined letrozole and gonadotrophins group than letrozole alone. There is no significant difference regarding follicular response and pregnancy rate in two age group
\end{abstract}

Introduction:

Throughout history, human societies were preoccupied by the process of procreation which is the major aspects of maintaining survival and perpetuation of the human race. Infertility is a well known cause of disharmony and disgrace among couples (mark of displeasure, multiple marriages among Muslims, grounds for divorce, and in the extremes, suicide), since time immemorial. The first written document regarding infertility was the Kahoun Papyrus (2200-1950 B.C.). 1, ${ }^{2}$ Bangladesh is a developing country with an exploding population of 158 million, a total fertility rate of 2.74 per thousand live births and a growth rate of $1.292 \%{ }^{3}$ In spite of that, there are a large number of couples who remain childless for years.

Prevalence of infertility throughout the world range from $10 \%-15 \%$ of couple. ${ }^{4,5}$ In a study of rural population of Bangladesh 3.2\% have primary infertility and 3\% have secondary infertility. Majority of infertile women (40\%) believed this to be their fate and $33 \%$ accused themselves for infertility. ${ }^{6}$

Prior to the 1960 s, infertility was almost exclusively a diagnostic statement Infertility treatment started 50

1. Asst. Prof, Dept of Gynae and Obs SSMC and Mitford Hospital

2. Junior Consultant (Infertility), Centre for Assisted Reproduction (CARE), BIRDEM

3. Prof and Honorary Chief Consultant, Dept of Obstetrics and Gynaecology, BIRDEM

4. Associate Prof, Dept of Gynae and Obs SSMC and Mitford Hospital

5. Prof, Dept. of Gynae and Obs, Kumudini Women's Medical College, Mirzapur, Tangail 
years ago with the use of exogenous gonadotrophins. ${ }^{7}$ The introduction of invitro fertilization \& embryo transfer (IVF-ET) in 1980s has made it possible for the couple to take advantage of this technology. ${ }^{7}$

Controlled ovarian hyper stimulation combined with IUI of capacitated sperm ( $\mathrm{COH}-\mathrm{IUI})$ has recently been used to treat a subset of couples infertile in the absence of mechanical compromise of the pelvic viscera, in whom no other efficacious treatment options exist $.8,9,10$. The major difference of $\mathrm{COH}$-IUI with IVF is that the normal fallopian tubes can be anticipated to function appropriately and no surgical procedure is required to collect oocytes. ${ }^{8,9,10}$ The benefit of IUI in male factor infertility has been accepted because IUI appears to result in higher pregnancy rates than natural intercourse or Intra Cervical Insemination (OR 2.20, 95\% Cl 1.43-3.39). ${ }^{11}$ In a review, Hammburg and Insler ${ }^{12}$ concluded that, taking into account efficacy, complication rates and cost of infertility treatment women with hypogonadotrophic hypogonadism or polycystic ovary syndrome (PCOS) should be offered acceptable methods of ovulation induction and that couples with 'unexplained' or 'multifactorial subfertility' should be exposed to controlled ovarian hyperstimulation with IUI (COH IUI) and only after the failure of these therapies should be offered invitro fertilization (IVF). ${ }^{12}$

For $\mathrm{COH}$ in IUI cycles, ovulation induction protocols are Clomiphene citrateCc, Aromatase inhibitor (Letrozole) alone or in combination with Gonadotrophins (hMG or rFSH), human chorionic Gonadotrophins (hCG); and the use of GnRH agonist or antagonist combined with Gonadotrophins. hCG is usually used at the end of the stimulation phase to achieve final maturation of the oocytes. ${ }^{13}$ The addition of IUI to Letrozole increase fecundity in couples with unexplained infertility or surgically treated endometriosis. ${ }^{14,15}$. Ovarian stimulation with letrozole is associated with acceptable pregnancy rates compared with gonadotrophin (cumulative pregnancy rate per couple: $24 \%$ vs. $36 \%$ ), ${ }^{16}$ with significant less cost, risk and patient inconvenience.

The combination of IUI with gonadotrophin and letrozole stimulation provides even better results than the combination of IUI with clomiphene. ${ }^{17}$

\section{Materials and Methods:}

This study was conducted at Centre for Assisted Reproduction (CARE), BIRDEM, Dhaka, during the period of $10^{\text {th }}$ May to $31^{\text {st }}$ December, 2010. All the infertility patients who were selected for IUI from CARE OPD BIRDEM were included in this study. Informed consent were taken from the couples and interviewed by a structured questionnaire

Inclusion criteria: The female patients with age $<40$ yrs, history of infertility for at least 2 yrs and with either of the following problems (unexplained infertility, polycystic ovarian syndrome, mild endometriosis) were included. Males with mild to moderate male factor sub fertility with total count (>10 million/ml), motility grade $\mathrm{A}(>10 \%)$, morphology $(>30 \% \mathrm{~N})$ and erectile dysfunction or hypospadias were included.

Exclusion criteria: Female with bilateral tubal block, severe endometriosis and no ovarian reserve, and male with sperm count <10 million / $\mathrm{ml}$ were excluded.

\section{Variables of the study:}

Input variable: Type and dose of ovulation inducing agent

Output variable: Follicular size, endometrial thickness, pregnancy outocme

Study Procedure: The patients were divided in to two groups. Group A: Female with young age $<30$ years, and Group B: Female with age $>30$ years.

\section{Superovulation Protocol:}

Both groups of patients were stimulated by Letrozole $7.5 \mathrm{mg}$ daily, started on D2 of the cycle and continued for 5 days. Patients were followed by this treatment for 3 cycles of IUI. Follicular response was assessed by TVS on D12 - 13, when at least two follicles attained $>18 \mathrm{~mm}$ size or single follicle $>21 \mathrm{~mm}$ size then it was considered satisfactory. This treatment was followed for 3 cycles. The patients who did not respond satisfactorily by 3 cycles of Letrozole were selected for combination of Letrozole and Gonadotrophins. These patients were stimulated by Inj. Gonadotrophins (rFSH $50 \mathrm{iu} / \mathrm{inj}$. hMG $75 \mathrm{iu}$ ) intra muscularly daily starting from D3 for 5-7 days + Letrozole 7.5 mg per day on $D_{2}-D_{6}$. Patients were followed up byTVS on D12/D13 (follicular size, number and endometrial thickness).In both regims when follicles attain satisfactory size, Inj. HCG (pregnyl/professy) 5000 IUI was given intra muscularly. A single IUI insemination was performed 36 hours after the Inj. of hCG .

Main outcome measure: Number of the follicle 2-3, Size of the follicle $>18 \mathrm{~mm}$ and Endometrial thickness $>8 \mathrm{~mm}$ was considered as satisfactory. 


\section{Definition of Pregnancy:}

Patients were asked to attend the OPD of CARE BIRDEM 15 days after the missed period. Positive pregnancy test result was defined as pregnancy. Pregnant patients were advised to come for regular antenatal checkup.

Data processing \& analysis: Result of the study was analyzed by standard statistical methods by using SPSS (Statistical Package for Social Science) software, version 16 (SPSS, Inc. Chicago, IL, USA) and Excel windows programme. The data was expressed as mean \pm SD and median (range) as appropriate. Student's unpaired t-test was used for calculating the difference between the groups. $\div 2$ (chi square) test was performed to determine the clustering of different variable of interest. $P$ value of less than 0.05 was considered statistically significant.

\section{Observation and result:}

Patients Characteristics

\begin{tabular}{|c|c|c|c|}
\hline Variables & Mean & SD & Percentage (\%) \\
\hline Age & 31.08 & 4.86 & \\
\hline Duration of infertility & 9.45 & 4.59 & \\
\hline \multicolumn{4}{|l|}{ Parity } \\
\hline \multicolumn{2}{|c|}{ Nulliparous (Primary Subfertility) } & & $77.92 \%$ (60 pts.) \\
\hline \multicolumn{2}{|c|}{ Multiparous(Secondary Subfertility) } & & $22.8 \%$ (17 pts.) \\
\hline \multicolumn{4}{|c|}{ Clinical Presentation } \\
\hline \multicolumn{2}{|l|}{ Oligo/anovulation } & & $74.02 \%$ (57 pts.) \\
\hline \multicolumn{2}{|l|}{ Hyperandrogenism } & & $14.28 \%$ (11 pts.) \\
\hline \multicolumn{2}{|l|}{ PCOS (TVS) } & & $55.05 \%$ (42 pts.) \\
\hline BMI (weight in $\mathrm{kg} / \mathrm{m} 2$ ) & 22.7 & 3.24 & \\
\hline
\end{tabular}

PCOS was detected by TVS and FSH: LH ratio altered in $48.05 \%$

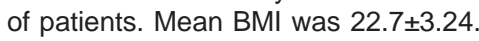

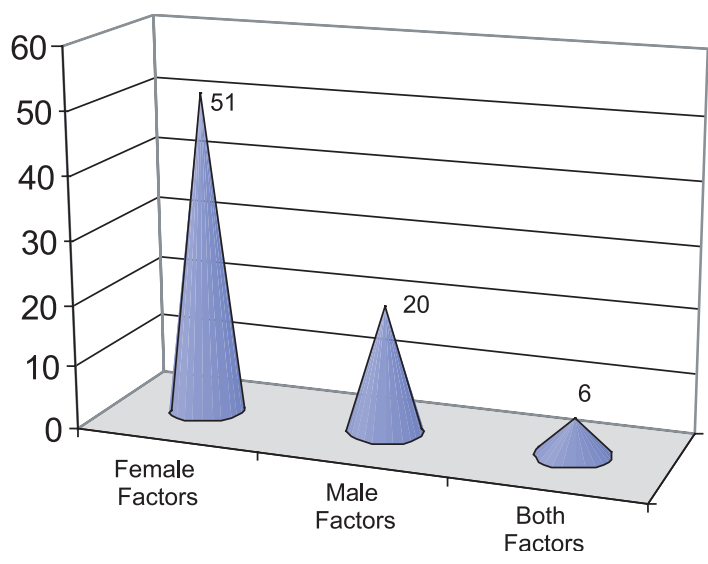

Factors responsible for infertility $(n=7)$

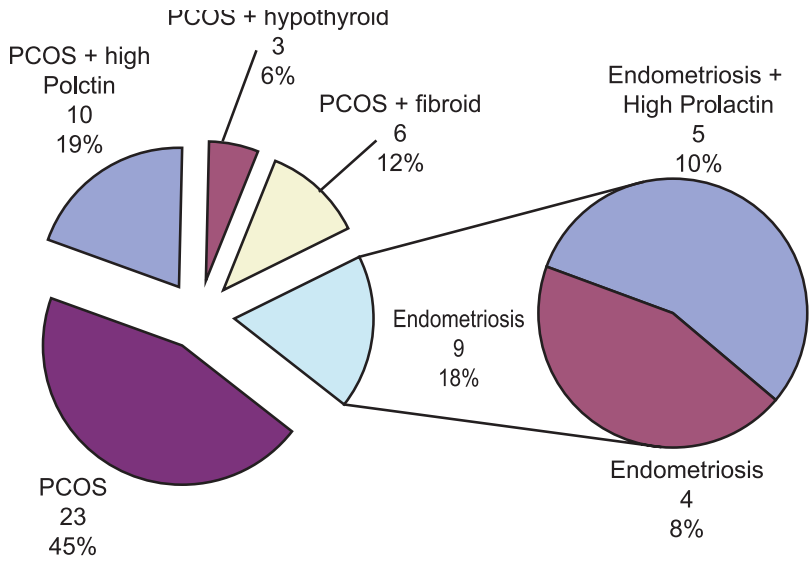

Female factors for infertility

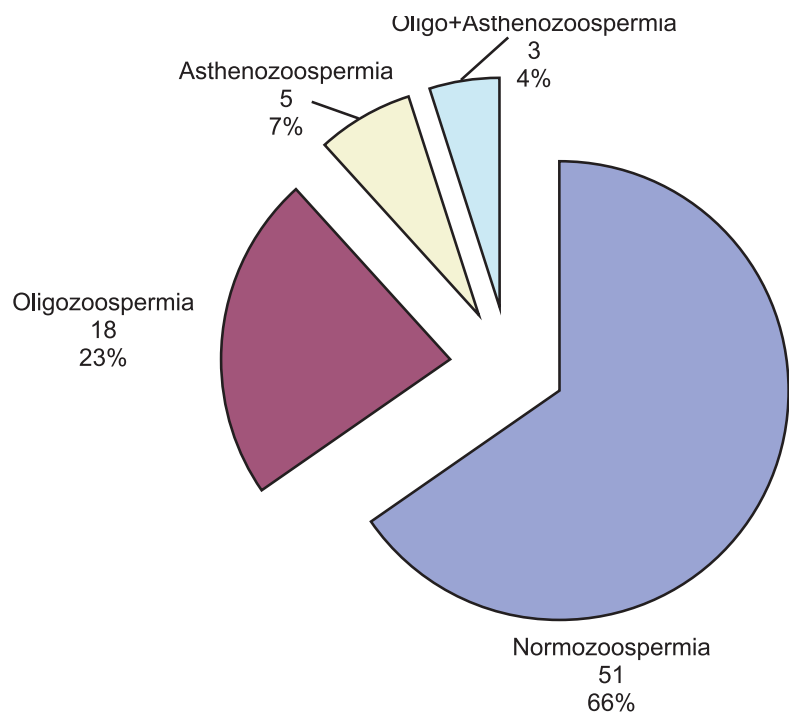

Semen analysis of husband on first visit

Among 77 pts, 35 (45\%) had normal FSH: LH ratio and 42 (55\%) had an altered ratio. Hyperprolactinaemia was present in 15 (20\%) patients. $3(4 \%)$ pts were hypothyroid. 77 patients were evaluated for tubal patency. Laparoscopy was used as a tool in $66(86 \%)$ patients and HSG in $11(14 \%)$ patients. Both tubes were patent in 59 (79\%) patients, and one tube was blocked in 18 (23\%) patients. During laparoscopy, ovarian drilling was done in PCOS patients. 
Table 2: Effect of letrozole and combined letrozole and gonadotrophins on follicular size

\begin{tabular}{|c|c|c|c|c|c|c|}
\hline Variables & \multicolumn{2}{|c|}{$\begin{array}{c}\text { Group A Letrozole }(\mathrm{n}=77) \\
\text { (cycle=231) }\end{array}$} & \multicolumn{4}{|c|}{$\begin{array}{l}\text { Group B Combined Letrozole and } \\
\text { Gonadotrophin }(n=65)(\text { cycle }=195)\end{array}$} \\
\hline No follicles & \multicolumn{2}{|c|}{38 pts $(49.36 \%)$} & \multicolumn{4}{|c|}{ Nil } \\
\hline pts with follicle size $10-14 \mathrm{~mm}$ & \multicolumn{2}{|c|}{14 pts (18.9 \%) } & \multicolumn{4}{|c|}{ Nil } \\
\hline pts with follicle size $14-18 \mathrm{~mm}$ & \multicolumn{2}{|c|}{13 pts $(16.88 \%)$} & \multicolumn{4}{|c|}{15 pts $(23.07 \%)$} \\
\hline pts with follicle size >18mm & \multicolumn{2}{|c|}{12 pts (15.58 \%) } & \multicolumn{4}{|c|}{50 pts $(76.93 \%)$} \\
\hline \multicolumn{7}{|c|}{$\begin{array}{c}\text { Table-III } \\
\text { Outcome in letrozole and combined letrozole and gonadotrophins }\end{array}$} \\
\hline Variables & $\begin{array}{c}\begin{array}{c}\text { Group A Letrozole } \\
(n=77) \\
(\text { cycle }=231)\end{array} \\
\end{array}$ & $\begin{array}{r}\text { Group B } \\
\text { and Go }\end{array}$ & $\begin{array}{l}\text { abined Letrozole } \\
\text { trophin }(n=65) \\
\text { e=195) }\end{array}$ & $\mathrm{t}$ & Ç2 & $\mathrm{P}$ \\
\hline Total no. of follicles & $1.28 \pm 0.94$ & & $7 \pm 0.87$ & 7.54 & & 0.0001 \\
\hline No follicles & 38 pts $(49.36 \%)$ & & nil & & & \\
\hline No. of follicles $10-14 \mathrm{~mm}$ & $2.08 \pm 1.68$ & & nil & & & \\
\hline No. of follicles $14-18 \mathrm{~mm}$ & $2.18 \pm 1.63$ & & $4 \pm 1.93$ & 2.48 & & 0.021 \\
\hline No. of follicles $>18 \mathrm{~mm}$ & (12 pts)3.54 \pm 1.63 & $(50$ & $3.44 \pm 1.93$ & 0.374 & & 0.71 \\
\hline Pre-treatment endometrial thickness & $5.93 \pm 1.06$ & & $3 \pm 1.07$ & 5.37 & & 0.0001 \\
\hline Post-treatment endometrial thickness & $7.89 \pm 0.93$ & & $1 \pm 1.4$ & 5.83 & & 0.0001 \\
\hline Duration of stimulation (days) & $12.2 \pm 1.3$ & & $3 \pm 2.8$ & & & \\
\hline Ovulation/cycle & 43/231 (18.6\%) & & $5(89.2 \%)$ & & 208.17 & 0.0001 \\
\hline
\end{tabular}

Mean of total no. of follicles (letrozole vs. combined letrozole and gonadotrophins group $1.28 \pm 0.94$ vs. $3.37 \pm 0.87$, $p$ value 0.0001 ). Endometrial thickness, (letrozole vs. combined letrozole and gonadotrophins $7.14 \pm 1.70 \mathrm{~mm}$ vs. $8.61 \pm 1.4 \mathrm{~mm}$, p value 0.0001 )

Comparison of follicular response between two age groups $(<30 \&>30)$

Table-IV

Induction used - Letrozole ( $7.5 \mathrm{mg} / \mathrm{d}$ for 5 days in a cycle)

\begin{tabular}{|c|c|c|c|c|c|}
\hline Variables & $<30$ yrs & $>30 \mathrm{yrs}$ & $\mathrm{t}$ & Ç2 & $\mathrm{P}$ \\
\hline No. of pt & 37 & 40 & & & \\
\hline No. of induction & 111 & 120 & & & \\
\hline No. of follicles $>18 \mathrm{~mm}$ in a cycle & 3.5 & 3.6 & 0.126 & & 0.9 \\
\hline Follicular size >18mm & 8 pts $(22 \%)$ & 5 pts $(13 \%)$ & 0.335 & & 0.74 \\
\hline Endometrial thickness (mm) & 8.52 & 8.7 & 0.556 & & 0.58 \\
\hline Ovulatory cycles (\%) & $28 / 111(25 \%)$ & $17 / 120(14.2 \%)$ & & 3.82 & 0.051 \\
\hline Pregnancy rate/cycle (\%) & 9/111 (08\%) & $3 / 120(03 \%)$ & & 2.63 & 0.11 \\
\hline Pregnancy patient rate & 9/37 (24\%) & $3 / 40(07 \%)$ & & 2.96 & 0.09 \\
\hline
\end{tabular}

In $>30$ yrs group, only $5(13 \%)$ patients had mature follicles, whereas in $<30$ yrs $8(22 \%)$ patients had mature follicles. $P$ value is 0.74 , which is not significant. 
Table-V

Induction used - Inj. FSH (50 IU or hMG, 75 IU for 5-7 days in a cycle)

\begin{tabular}{|c|c|c|c|c|c|}
\hline Variables & $<30$ yrs & $>30$ yrs & t test & Ç2 & $P$ \\
\hline No. of pt & 30 & 35 & & & \\
\hline No. of induction & 90 & 105 & & & \\
\hline No. of follicles $>18 \mathrm{~mm}$ in a cycle & $3.6 \pm 1.95$ & $3.3 \pm 1.94$ & 0.475 & & 0.64 \\
\hline Follicular size $>18 \mathrm{~mm}$ & 20 pts (66\%) & 30 pts (88\%) & 1.31 & & 0.2 \\
\hline Endometrial thickness(mm) & 8.52 & 8.7 & 0.693 & & 0.49 \\
\hline Ovulatory cycles (\%) & $20 / 90(23 \%)$ & $31 / 105(30 \%)$ & & 0.99 & 0.32 \\
\hline Pregnancy rate/cycle (\%) & $16 / 90(17 \%)$ & $12 / 105(12 \%)$ & & 1.11 & 0.29 \\
\hline Pregnancy patient rate & $16 / 30(54 \%)$ & $12 / 35(34 \%)$ & & 1.68 & 0.19 \\
\hline
\end{tabular}

In $>30$ yrs age group, 30 (88\%) patients had mature follicles, whereas in $<30$ yrs age group, $20(66 \%)$ patients had mature follicles.P is 0.2 .

Table-VI

Comparison of pregnancy outcome between letrozole and combined groups

\begin{tabular}{|c|c|c|c|c|c|}
\hline Variables & $\begin{array}{c}\text { Group A Letrozole }(n=77) \\
\text { cycle }=231)\end{array}$ & $\begin{array}{c}\text { Group B Comb Letr. \& } \\
\text { Gonadotrophins }(n=65) \\
\text { (cycle=195) }\end{array}$ & $\mathrm{t}$ & $\S^{2}$ & $P$ value \\
\hline Duration of stimulation (days) & $12.2 \pm 1.3$ & $10.3 \pm 2.8$ & & & \\
\hline Ovulation cycle & $43 / 231(18.6 \%)$ & 174/195 (89.2\%) & & 208.17 & 0.0001 \\
\hline Pregnancy cycle & $12 / 231(5.19 \%)$ & 28/195 (14.3\%) & & 9.39 & 0.0022 \\
\hline Pregnancy patient rate & $12 / 77(16 \%)$ & $28 / 65(43 \%)$ & & 11.84 & 0.0006 \\
\hline
\end{tabular}

Discussion:

$\mathrm{COH}$ and IUI are common treatments for couples with unexplained infertility and those with infertility associated with specific diagnoses in which no pregnancy has occurred despite standard directed treatments. Total 77 patients were selected for IUI in our centre CARE BIRDEM. All patients had some sort of past infertility treatment, at various levels from various parts of the country. In the present study, mean age of female patient was $31 \pm 4.8 \mathrm{yrs}$. The mean duration of infertility was $9.5 \pm 4.6 \mathrm{yrs}$. By letrozole there was no follicular development in 38 (49.36\%) patients. Mature follicles developed in only 12 (15.58\%) patients. On the contrary by combined gonadotrophins and letrozole mature follicles appeared in 50 (76.93\%) patients. Average duration of cycle (letrozole vs. combined letrozole and gonadotrophins) was $12.2 \pm 1.3$ vs.10.3 \pm 2.8 days. Ovulation per cycle (letrozole vs. combined letrozole and gonadotrophins) was $18.6 \%$ vs. $89.2 \%$. The $p$ value is 0.0001 , which is significant. , Endometrial thickness $>7 \mathrm{~mm}$ (letrozole vs. combined letrozole and gonadotrophins) was 37 (48\%) pts vs. $58(90 \%)$ pts. P value is 0.00013 , which is significant.
$12 / 231$ cycles showed pregnancy in letrozole group, whereas 28/195 cycles showed pregnancy in combined group. Pregnancy per cycle is $5.1 \%$ in letrozole group and $14.3 \%$ in combined group. Pregnancy patient rate is $\{12 / 77$ (16\%) vs. $28 / 65$ $(43 \%)\}$. $P$ value is 0.0006 which is significant.

In this study, letrozole was associated with fewer developing and mature follicle, which is not comparable with the study by Mitwally \& Casper ${ }^{14}$, Al-Omari et al. ${ }^{18}$ who showed ovulatory rate by letrozole was $75 \%$ and $87.5 \%$, respectively. Another study by Mitwally 15 showed that treatment with letrozole+ FSH required less amount of FSH (616 IU vs. $1590 \mathrm{IU}$ ) than FSH alone, and the number of follicle $>18 \mathrm{~mm}$ was higher, in combined letrozole and FSH group (3.3 vs. 1.9, respectively) which is consistent with our study (3.37 \pm 0.87 vs. $1.28 \pm 0.94$ ).

Another study comparing 3 treatment protocols letrozole+ $\mathrm{FSH}, \mathrm{CC}+\mathrm{FSH}$ and $\mathrm{FSH}$ only in patients with unexplained infertility undergoing $\mathrm{COH}$ and IUI by Mitwally ${ }^{14}$ showed that the total FSH dose was lowest in letrozole+ FSH group, but the endometrial thickness was highest in the letrozole+ FSH group 
$(9 \mathrm{~mm})$. The clinical pregnancy rate was highest in letrozole+ FSH group (22.2\%) which is comparable with our study, where pregnancy rate is $14.3 \%$ in combined letrozole and gonadotrophins group. The results of this study suggest that the addition of letrozole to $\mathrm{FSH}$ can reduce the dose of $\mathrm{FSH}$ and medication expense without detrimental effects on endometrial thickness and pregnancy.

In another study by Rumste ${ }^{19}$ regarding COS and IUI in unexplained infertility showed that $32 \%$ of the couples conceived in four treatment cycles of COSIUI which is within the range of $20-33 \%$ reported by others (Guzick et al. ${ }^{20}$ ). In other literatures by Crosignani ${ }^{21}$, Guzick et al. ${ }^{20}$, Dickey et al. ${ }^{22}$ pregnancy rate per cycle has been reported to vary from $9-20 \%$. It has been shown that increasing maternal age, longer duration of non-conception and a poor semen quality have a negative impact on the pregnancy rate in couples receiving COS-IUI (Dickey et al. $2002^{22}$ ). Multifollicular growth contains the risk of multiple pregnancies. Another study by Sarah Healey et $\mathrm{al}^{23}$ about the effect of letrozole and gonadotrophins in IUI patients showed that they required less gonadotrophin administration (median difference, $300 \mathrm{IU}$ at $95 \% \mathrm{CI}, 225-375 \mathrm{IU}$ ) and had a thinner endometrium, ( $\mathrm{m}$ df $1 \mathrm{~mm}$ ), developed more follicles $>14 \mathrm{~mm}$ ( $\mathrm{m} \mathrm{df}, 1$ follicle $95 \% \mathrm{Cl}$, 1-2 follicle). But the pregnancy rate did not differ between patients using gonadotrophins alone and those using gonadotrophins+ letrozole (20.9\% vs. $21.6 \%)$. Between two age groups, in $>30$ yrs group, only 5 $(13 \%)$ patients had mature follicles, whereas in $<30$ yrs $8(22 \%)$ patients had mature follicles. $P$ value is 0.74 , which is not significant. Ovulatory cycle was $28 / 111$ cycles, in $<30$ yrs age group, and 17/120 cycles, in $>30$ yrs age group. $P$ is 0.051 which is not significant. Pregnancy rate/cycle was $9 / 111$ cycles in $<30$ yrs age group, and $3 / 120$ cycles in $>30$ yrs age group. $P$ value is 0.11 , which is not significant. Thus there is no statistical significance in follicular response and pregnancy rate by giving letrozole alone in these two groups.

65 patients were poor responders to letrozole were treated with combined letrozole and gonadotrophins. In $>30$ yrs age group, 31 (86\%) out of 35 patients had mature follicles, whereas in $<30$ yrs age group, 20 $(66 \%)$ out of 30 patients had mature follicles. $P$ value is 0.2 , which is not significant. Therefore there is no significant difference in follicular response between two age groups by gonadotrophins. But $>30$ yrs age group required more doses of gonadotrophin (avg of 5-7 doses) than $<30$ yrs age group.

There is no known national or international study regarding the follicular response of different age groups. There is no national study regarding the controlled ovarian stimulation and IUI, with which to compare.

\section{Conclusion:}

Infertility definitely has some tragic dimensions in the family life of many couples. The cause of infertility varies from country to country and in different social groups. Endometriosis, PCOS and male factor are common cause in developed countries, whereas STDs are the main cause in African countries. Incidence of tubal occlusion in our country can be prevented by safe delivery, safe abortion and post abortal care and increasing the awareness about infertility. For PCOS resistant to clomiphene or letrozole, mild endometriosis, unexplained infertility and also mild to moderate male factor infertility, the combination of superovulation and intrauterine insemination is an effective measure for achieving pregnancy. Moreover the effects of superovulation and IUI appear to be independent and additive. IUI and $\mathrm{COH}$ is relatively simple, non-invasive, inexpensive and effective measure. It can be provided more easily to more infertile couples in office practices and general hospitals than can the more specialized techniques such as IVF. However, careful selection of patients is required for successful IUI. Studies by Goverde et al. ${ }^{24}$ on cost effectiveness of IUI and $\mathrm{COH}$ showed that it was as effective as IVF in achieving pregnancy (31\% vs. $33 \%$ ) and was more cost effective than IVF. So these two groups of patients should be counselled that IUI and $\mathrm{COH}$ is their preferable method, and there is least chance of complications like hyperstimulation syndrome, multiple pregnancy. Many infertile couples of today may have new hope for future days that science will continue to unravel the mysteries of procreation and with the discovery of each mystery more infertile couples will enjoy the fulfilment of bearing a child.

\section{Reference:}

1. Griffith FL. The Petrie (Kahoun) Papyrus. London, 1898

2. Griffith FL. A medical Papyrus from Egypt. Br Med J 1893; 1: 1172-1174 
3. Bangladesh demographic Data Sheet and Survey-2007

4. Whiteford L.M, Gonzalez L. Stigma: the hidden burden of infertility. Soc Sc Med 1995; 40:27-36

5. Downey J, Yingling S, McKinney M, Husami N. Mood disorders, psychiatric symptoms and distress in women presenting for infertility evaluation. Fertil Steril 1989; 52: 425-432.

6. Bangladesh Institute of Research in Promotion of Essential and Reproductive Health and Technologies (BIRPERHT). Assessment of reproductive health care and review services provided at the level of thana, union and villages, August-1997 Issue, Vol-5.

7. Gemzell CA, Diczfalusy E, Tillinger G. Clinical effect of human pituitary follicle stimulating hormone (FSH). J Clin Endocrinol Metab 1958; 18(12): 1333-48

8. Dodson WC, Whitesides DB, Hughes CL, et al. Superovulation with washed intra-uterine insemination in the treatment of infertility: A possible alternative to gamete intra fallopian transfer and IVF. Fertil Steril 1987; 48: 441- 445

9. Dodson WC, Haney AF. Controlled ovarian hyperstimulation and intra-uterine insemination for treatment of infertility. Fertil Steril 1991; 55:457467.

10. Corson GH, Kemmann E. The role of superovulation with menotropins in ovulatory infertility: a review. Fertil Steril 1991; 55: 468477

11. Ford WC, Mathur RS, Hull MG. Intra uterine insemination: is it an effective treatment for male factor infertility? Baillieres clin obstet Gynaecol 1997: 11: $691-710$

12. Hamburg $R$, Insler V. Ovulation induction in perspective. Hum Reprod update 2002; 8: 449 462.

13. Brinsden PR and Dickey RP. An overview of intrauterine insemination and ovulation induction. Manual of intra uterine insemination and ovulation induction. Cambridge university press - 2010; ch - 1: p-1-6.

14. Mitwally MF, Casper RF, Potential of aromatase inhibitors for ovulation and super ovulation induction in infertile women. Drugs 2006; 66 (17): $2149-60$.

15. Mitwally MF, Casper RF, Aromatase inhibition improves ovarian response to follicle stimulating hormone in poor responders. Fertil steril 2002. April; 77 (4): 776 - 80.

16. Gregoriou O, Vlahos NF, Konidaris S et al. Randomized control trial comparing superovulation with letrozole vs. rFSH combined with IUI for couples with unexplained infertility who had failed clomiphene citrate stimulation and IUI. Vol. 90, Issue 3, 678-683, Sep 2008

17. Ganesh A, Goswami SK, Chattopadhyay, Chaudhury K, Chakrabarty BM. Comparison of letrozole with continuous gonadotrophins and clomiphene gonadotrophin combination for ovulation induction in 1387 PCOS women after clomiphene citrate failure: A randomized prospective clinical trial. J of asst. repro gent. 2/09 January 26(1): 19-24.

18. Al-Omari WR, Sulaiman W, Al-Hadithi. Comparison of two aromatase inhibitors in women with PCOS. Intern J Gynaecol Obstet 2004; 85 : 289-291

19. Rumste MME, Hartog JE, Dum oulin JCM, etal. Is controlled ovarian stimulation in intra uterine insemination an acceptable therapy in couples with unexplained non conception in the perspective of multiple pregnancies? Hum. Reprod ; Vol-21: No - 3, P-701, 2006.

20. Guzick DS, Carson SA, et al (1999). Efficacy of super ovulation and intra uterine insemination in the treatment of infertility. National cooperative Reproductive Medicine network. New Engl. J. Med 340. $177-183$.

21. Crosignani PG, Walters DE and Soliani A (1991), The ESHRE multicentric trial on the treatment of unexplained infertility: A preliminary report. Hum Reprod 6, 953 - 958.

22. Dickey RP, Taylor SN, LU PY, et al. Risk factors for high order multiple pregnancy and multiple birth after controlled ovarian hyper stimulation; results of 4062 intrauterine insemination cycles. Fertil steril 2005; 83: $671-83$.

23. Sarah Healey, Seang LT, Togas T, et al. Effects of letrozole on superovulation with gonadotrophins in women undergoing IUI. Fertil Steril; 80: 1325-1329. Dec 2003

24. Goverde AG, McDonnell J, Vermeiden JP. IUI or IVF in idiopathic subfertility: A randomized trial and cost effectiveness analysis. Lancet 2000; 355: 13-18. 